\title{
Utility of repeating bone marrow biopsy for confirmation of complete response in multiple myeloma
}

\author{
Marcella A. Tschautscher ${ }^{1}$, Dragan Jevremovic ${ }^{2}$, Francis K. Buadi ${ }^{1}$, Martha Q. Lacy', Morie A. Gertz ${ }^{1}$, Angela Dispenzieri ${ }^{1}$, \\ Prashant Kapoor', David Dingli', Lisa Hwa', Amie Fonder', Miriam Hobbs' ${ }^{1}$, Suzanne Hayman', John Lust',

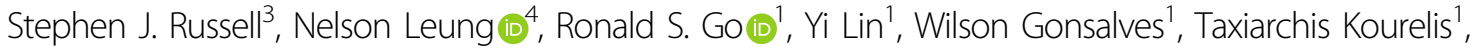 \\ Rahma Warsame', Robert A. Kyle', S. Vincent Rajkumar (1) and Shaji Kumar (10 ${ }^{1}$
}

\section{Introduction}

Achievement of complete response (CR) in multiple myeloma (MM) has been defined by the International Myeloma Working Group (IMWG) as concurrent demonstration of disappearance of monoclonal protein in the serum and urine with negative immunofixation (IFE), in addition to a bone marrow biopsy demonstrating $<5 \%$ bone marrow plasma cells (BMPC) $)^{1,2}$. With advancements in myeloma-directed therapies and increasing $\mathrm{CR}$ rates, it is imperative that accurate and uniform application of $C R$ criteria be utilized, especially with increased incorporation of minimal residual disease assessment ${ }^{3,4}$. While obtaining serum and urine samples for monoclonal protein analyses are simple, bone marrow biopsies are associated with pain, are inconvenient, and are burdensome to patients.

We are occasionally confronted with the scenario of the bone marrow fulfilling the criterion for $\mathrm{CR}(<5 \% \mathrm{PCs})$ with simultaneous positive serum or urine immunofixation. In these circumstances, a repeat bone marrow biopsy would be required at the time of serum and urine monoclonal protein disappearance, subjecting patients to another painful and uncomfortable procedure that is unlikely to yield different results or influence clinical decision making. We, therefore, investigated the utility of repeating a BM biopsy at the time of serum and urine IFE negativity in myeloma patients undergoing autologous

Correspondence: Shaji Kumar (kumar.shaji@mayo.edu)

'Division of Hematology, Mayo Clinic, Rochester, MN, USA

${ }^{2}$ Division of Hematopathology, Mayo Clinic, Rochester, MN, USA

Full list of author information is available at the end of the article stem cell transplant (ASCT) with prior marrow demonstrating $<5 \%$ PCs.

\section{Patients and methods}

A retrospective cohort study was conducted on all myeloma patients seen at Mayo Clinic who underwent an autologous stem cell transplant between 1998 and 2016 . Patients included in the analysis had to have a pretransplant BM biopsy demonstrating < $5 \%$ PCs with concomitant serum and urine IFE studies and post-transplant bone marrow biopsy, serum, and urine IFE studies available for analysis. Frequency and degree of BMPC clonality were recorded in the pre-transplant BM biopsy. Serum and urine immunofixation status, collected within 30 days of the pre-transplant bone marrow biopsy was recorded as positive or negative. Among patients who had a positive pre-transplant serum or urine IFE, post-transplant data (day 100 after SCT) including BMPC percentage, serum IFE, and urine IFE were recorded, with all IFE collections occurring within 30 days from the bone marrow biopsy. We then examined the proportion of patients in whom the serum and urine IFE became negative and its association with BMPC percentages. All statistical analyses, storage of data, and image generation were performed using the JMP 14.1.0 statistical package (SAS Institute Inc., Cary, NC). Approval for this study was obtained from the Mayo Clinic IRB and informed consent was obtained from all patients for review of their medical records.

\section{Results}

The median time from the pre-transplant bone marrow biopsy to date of transplant was 0.53 months 
(0.2-5 months). We identified 277 patients in our database with pre-transplant IFE positivity and pre-transplant BMPC $<5 \%$ with post-transplant data available for analysis. Among these patients, 179 (64.6\%) patients had detectable clonal plasma cells in the pre-transplant BM biopsy, with a median clonal PC percentage of $0.55 \%$ (0.1\%-29\%). Following the transplant, 116/277 (42\%) patients were found to meet IFE criteria for CR (i.e. IFE negative in both serum and urine) while 161 (58\%) patients remained IFE positive post-transplant in either the serum or urine (VGPR).

A repeat marrow examination was done at a median of 3.3 months (1.7-4.9 months) from transplant. Among the patients with negative post-transplant serum and urine IFE $(n=116), 98 \%$ had unchanged BMPC percentage post-transplant $(\mathrm{BMPC}<5 \%)$ and the remaining 2 patients demonstrated BMPC compositions of $15 \%$ and $7 \%$, both of which had $6 \%$ clonal plasma cells. In contrast, among the cohort of patients who were IFE positive posttransplant, $14(8.7 \%)$ demonstrated an increase in PC composition in their post-transplant bone marrow biopsies, with a median BMPC cellularity of $7.5 \%(5-80 \%)$ among which 12 had detectable clonal plasma cells with a median of 5.6\% (0.2-41.2\%), (Fig. 1). The remaining 94\% of patients had unchanged BMPC composition (Fig. 1).

\section{Discussion}

Based on current IMWG criteria for confirming CR in myeloma, a patient must demonstrate serum and urine

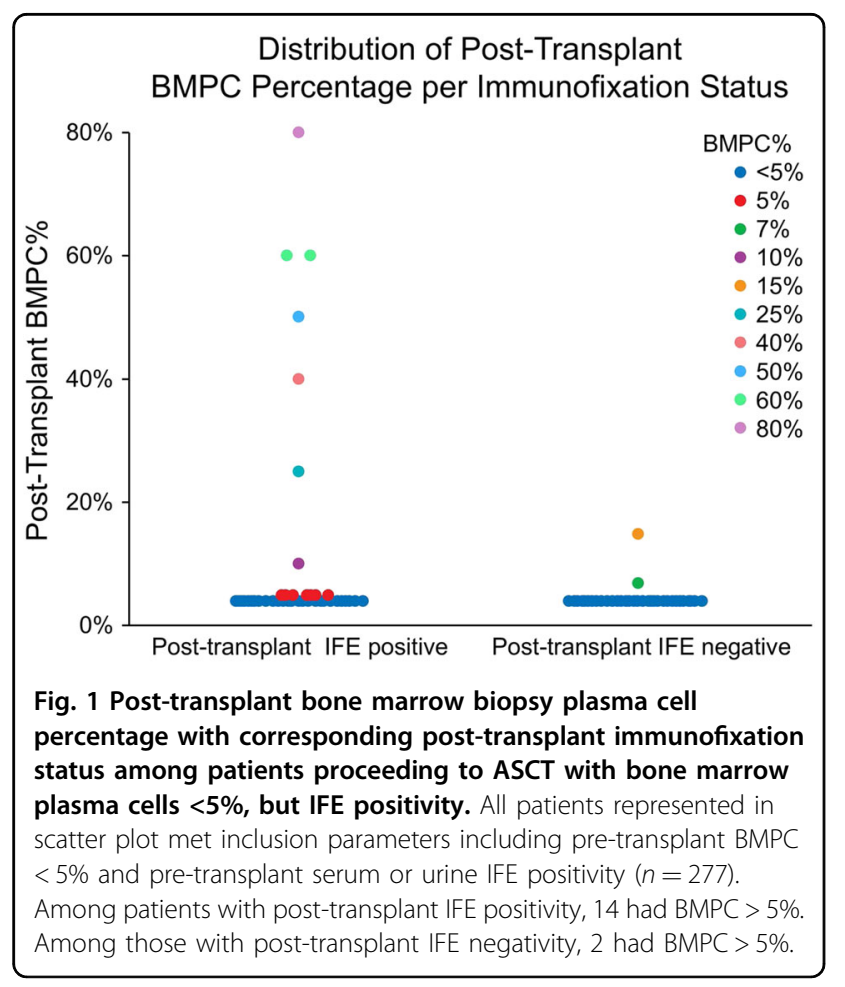

IFE negativity with concomitant bone marrow findings of $<5 \%$ plasma cells ${ }^{1}$. While the timing in concordance of these parameters allows for only one bone marrow biopsy, we are occasionally confronted with conflicting results, specifically with a bone marrow biopsy fulfilling criteria for CR with contradictory peripheral studies. In these cases, IMWG guidelines would suggest repeating a bone marrow biopsy at the time when both serum and urine IFE become negative, thus subjecting the patient to another distressing and painful procedure ${ }^{1}$.

The results of our study demonstrate that in $98 \%$ of patients whose bone marrow biopsy met criteria for $\mathrm{CR}$ before immunofixation studies (negative urine and serum IFE), there was no difference in the BMPC percentage with a repeat biopsy after ASCT. One explanation for this phenomenon is the difference in the monoclonal protein clearance kinetics and BMPC composition, with a delay in disappearance of $M$ protein relative to marrow disease clearance $^{5}$. Among the remaining patients with available post-transplant serum and urine IFE not otherwise meeting criteria for $\mathrm{CR}, 14$ (8.7\%) demonstrated an increase in the composition of BMPC from $<5 \%$ pretransplant. This finding could be explained by myeloma's patchy marrow involvement which is reflected in the initial, falsely reassuring bone marrow biopsy with true disease activity captured upon subsequent marrow sampling $^{6}$. This rationale is supported by the presence of clonal plasma cells in the post-transplant BM biopsy in this cohort, detected in 12 of 14 patients. Similarly, the observed inconsistency could also be due to an aggressive disease phenotype with progression occurring near day 100, however in these settings serum and/or urine IFE were in accordance with BM findings. The limitation in relying solely on the bone marrow biopsy is further supported with the finding of positive immunofixation either in the serum, urine, or both in these clinical settings. This reinforces the need and utility of all three parameters to accurately confirm a state of CR, however not necessarily simultaneously.

One limitation of our study is this analysis only evaluated IMWG criteria for CR and not stringent complete response $(\mathrm{sCR})$, which requires serum free light chain assessment and the absence of clonal plasma cells via immunohistochemistry. Secondly, we do not provide details regarding the status of soft tissue plasmacytomas via imaging to correlate with our findings. Finally, while $64 \%$ of patients had detectable clonal plasma cells in the pre-transplant biopsy, in general the proportion of clonal PCs in these samples were found at low prevalence (median $0.55 \%$ ), the relevance of which has been shown to correlate with improvement in clinical outcomes, especially if present at $<5 \%$.

Our findings suggest that in the setting where premyeloma directed therapy serum or urine IFE lag in 
fulfilling $\mathrm{CR}$ criteria with $\mathrm{BMPC}<5 \%$, repeating a bone marrow biopsy after treatment is unnecessary and unlikely to provide any additional information and influence approach to care outside of the clinical trial setting. This would save many myeloma patients from unneeded biopsies if added to the current IMWG complete response criteria.

\section{Acknowledgements}

This study was funded by The Mayo Clinic Hematological Malignancies Program.

\section{Author details}

${ }^{1}$ Division of Hematology, Mayo Clinic, Rochester, MN, USA. ${ }^{2}$ Division of Hematopathology, Mayo Clinic, Rochester, MN, USA. ${ }^{3}$ Division of Hematology and Molecular Medicine, Mayo Clinic, Rochester, MN, USA. ${ }^{4}$ Division of Nephrology and Hypertension, Department of Internal Medicine, Mayo Clinic, Rochester, MN, USA

\section{Author contributions}

S.K.K. and M.A.T. designed the study, collected and analyzed the data, and wrote the paper. D.J., S.V.R., A.D., M.Q.L., M.A.G., F.K.B, D.D., L.H., A.F., M.H., S.R.H, J. A.L., S.J.R., N.L., P.K., R.S.G., Y.L., W.I.G., T.K., R.W., and R.A.K. were involved in patient management, revised the paper, and approved the final version of the paper.

\section{Competing interests}

The authors declare no competing interests.

\section{Publisher's note}

Springer Nature remains neutral with regard to jurisdictional claims in published maps and institutional affiliations.

Received: 20 July 2020 Revised: 14 September 2020 Accepted: 18 September 2020

Published online: 02 October 2020

\section{References}

1. Kumar, S. et al. International Myeloma Working Group consensus criteria for response and minimal residual disease assessment in multiple myeloma. The Lancet Oncology. 17, e328-e346 (2016).

2. Chee, C. E. et al. The importance of bone marrow examination in determining complete response to therapy in patients with multiple myeloma. Blood. 114 2617-2618 (2009).

3. van de Velde, H. J. K. et al. Complete response correlates with long-term survival and progression-free survival in high-dose therapy in multiple myeloma. Haematologica. 92, 1399-1406 (2007).

4. Paiva, B., van Dongen, J. J. M. \& Orfao, A. New criteria for response assessment: role of minimal residual disease in multiple myeloma. Blood. 125, 3059-3068 (2015).

5. Paiva, B. et al. Comparison of immunofixation, serum free light chain, and immunophenotyping for response evaluation and prognostication in multiple myeloma. 29, J.Clin. Oncol. 1627-1633 (2011)

6. Waldschmidt, J. M., Anand, P., Knoechel, B. \& Lohr, J. G. Comprehensive characterization of circulating and bone marrow-derived multiple myeloma cells at minimal residual disease. Semin Hematol. 55, 33-37 (2018).

7. Tschautscher, M. A. et al. Prognostic value of minimal residual disease and polyclonal plasma cells in myeloma patients achieving a complete response to therapy. Am J Hematol. 94, 751-756 (2019). 